Pesq. Vet. Bras. 30(10):833-838, outubro 2010

\title{
Doença do armazenamento lisossomal induzida pelo consumo de Sida carpinifolia em bovinos do Rio Grande do Sul ${ }^{1}$
}

\author{
Pedro M.O. Pedroso², Luiz G.S. de Oliveira3 ${ }^{3}$, Cláudio E.F. Cruz³ ${ }^{3}$ Mauro P. \\ Soares $^{4}$, Laura R.B. Barreto ${ }^{5}$ e David Driemeier ${ }^{3^{*}}$
}

\begin{abstract}
Pedroso P.M.O., Oliveira L.G.S., Cruz C.E.F., Soares M.P., Barreto L.R.B. \& Driemeier D. 2010. [Lysosomal storage disease caused by Sida carpinifolia in cattle in Rio Grande do Sul.] Doença do armazenamento lisossomal induzida pelo consumo de Sida carpinifolia em bovinos do Rio Grande do Sul. Pesquisa Veterinária Brasileira 30(10):833-838. Setor de Patologia Veterinária, Faculdade de Veterinária, Universidade Federal do Rio Grande do Sul, Av. Bento Gonçalves 9090, Porto Alegre, RS 91540-000, Brazil. E-mail: davetpat@ ufrgs.br

This paper reports the natural poisoning by Sida carpinifolia (guanxuma, chá-da-índia) in cattle in Rio Grande do Sul, Brazil. Five cattle were affected in the period 2001-2008. Clinical signs included weight loss, incoordination, walking difficulty, generalized tremors, frequent falls, and death. Microscopically, the main changes were vacuolation of Purkinje neurons in the cerebellum, pancreatic acinar cells, and thyroid follicular cells. Transmission electron microscopy revealed vacuoles bordered by membrane containing finely granular material. Lectin histochemistry showed positive staining in neurons with the lectins Concanavalia ensiformis (Con-A), Triticum vulgaris (WGA), and Succinyl Triticum vulgaris (sWGA).

INDEX TERMS: Poisonous plants, Sida carpinifolia, Malvaceae, plant poisoning, cattle, lysosomal storage disease, lectin histochemistry, transmission electron microscopy.
\end{abstract}

RESUMO.- Relata-se a intoxicação natural por Sida carpinifolia (guanxuma, chá-da-índia) em bovinos no Rio Grande do Sul. Foram afetados cinco bovinos no período 20012008. O quadro clínico foi caracterizado por emagrecimento, incoordenação, dificuldade de locomoção, tremores generalizados, quedas frequentes e morte. Microscopicamente, as principais alterações foram vacuolização dos

\footnotetext{
${ }^{1}$ Recebido em 17 de maio de 2010.

Aceito para publicação em 5 de julho de 2010.

Parte da tese de doutorado do primeiro autor.

2 Programa de Pós-Graduação em Ciências Veterinárias, Universidade Federal do Rio Grande do Sul (UFRGS), Av. Bento Gonçalves 9090, Porto Alegre, RS 95320-000, Brasil.

${ }^{3}$ Setor de Patologia Veterinária (SPV), Departamento de Patologia Clínica Veterinária, Faculdade de Veterinária, UFRGS, Porto Alegre, RS 95320-000. “Autor para correspondência: davetpat@ufrgs.br

${ }^{4}$ Laboratório Regional de Diagnóstico, Faculdade de Veterinária, Universidade Federal de Pelotas (UFPel), Campus Universitário $\mathrm{s} / \mathrm{n}$, Pelotas, RS 96010-900, Brasil.

${ }^{5}$ Médica Veterinária, Secretaria Municipal da Agricultura, Rod. TF 010, 10 km 1, Centro, Triunfo, RS 95840-000, Brasil.
}

neurônios de Purkinje do cerebelo, das células acinares do pâncreas e das células foliculares da tireoide. A microscopia eletrônica evidenciou vacúolos com conteúdo finamente granulado e delimitado por membrana. Na lectinahistoquímica, observou-se marcação em neurônios com as lectinas Concanavalia ensiformis (Con-A), Triticum vulgaris (WGA) e Succinyl Triticum vulgaris (sWGA).

TERMOS DE INDEXAÇÃO: Plantas tóxicas, Sida carpinifolia, Malvaceae, bovinos, intoxicação por planta, doença do armazenamento lisossomal, lectina-histoquímica, microscopia eletrônica de transmissão.

\section{INTRODUÇÃO}

Sida carpinifolia L.f. (Malvaceae) ou Sida acuta var. carpinifolia (L.f) K.Schum, popularmente conhecida como guanxuma, vassourinha, malva-brava e relógio-de-vaqueiro é planta nativa do Brasil, onde ocorre em quase todo o território nacional. A planta é invasora de lavouras e pastagens, principalmente em solos mais argilosos, compactados e ricos em matéria orgânica (Kissmann \& Groth 2000, Lorenzi 2000). No Rio Grande do 
Sul, onde é vulgarmente conhecida como chá-da-india ou chádo-reino, a planta está disseminada, especialmente em áreas sombreadas e litorâneas. Nesse estado, a intoxicação foi diagnosticada, pela primeira vez, em caprinos (Driemeier et al. 2000), subsequentemente, em pôneis (Loretti et al. 2003), ovinos (Seitz et al. 2005a) e cervo dama (Dama dama) (Pedroso et al. 2009). A doença também foi identificada em Santa Catarina, onde foram afetados bovinos (Furlan et al. 2009) e, em São Paulo, onde caprinos e ovinos se intoxicaram (Godoy et al. 2005, Paganini Filho et al. 2008). Experimentalmente, a doença foi reproduzida em caprinos (Colodel et al. 2002a), ovinos (Seitz et al. 2005b) e bovinos (Furlan et al. 2008).

Os principais sinais clínicos da intoxicação incluem incoordenação, ataxia com dismetria, tremores de cabeça e pescoço, além de quedas frequentes (Colodel et al. 2002a, Furlan et al. 2008). Na necropsia, não são observadas alterações macroscópicas significativas (Colodel et al. 2002a, Seitz et al. 2005a, Furlan et al. 2008). As principais alterações microscópicas se caracterizam por distensão e vacuolização dos neurônios de Purkinje do cerebelo, núcleos do tronco encefálico, epitélio dos ácinos pancreáticos, células foliculares da tireoide, epitélio de túbulos renais, hepatócitos e macrófagos de órgãos linfóides (Colodel et al. 2002a, Seitz et al. 2005a, Furlan et al. 2008, Furlan et al. 2009).

O alcalóide indolizidínico 1,2,8-triol, denominado swainsonina e identificado em S. carpinifolia (Colodel et al. 2002b), inibe as enzimas $\alpha$-manosidase lisossomal e $\alpha$-manosidase II do complexo de Golgi e, consequentemente, induz a doença de depósito lisossomal (DDL) (Agamanolis 1995, Moremen 2002) que afeta particularmente neurônios (Molyneux \& James 1982, Stegelmeier et al. 1995). A detecção de oligossacarídeos na urina de caprinos intoxicados experimentalmente com Sida carpinifolia foi indicada como ferramenta diagnóstica para animais expostos ou intoxicados pela planta (Bedin et al. 2009). Doenças indistinguíveis têm sido associadas com consumo de Astragalus spp. e Oxytropis spp. nos Estados Unidos (Stegelmeier et al. 1995, Pfister et al. 2003), Swainsona spp. na Austrália (Colegate et al. 1979), Ipomoea spp. no Sudão (Damir et al. 1987), Moçambique (Balogh et al. 1999), Argentina (Armesto et al. 2004, Ríos et al. 2008) e Brasil (Barbosa et al. 2006, Antoniassi et al. 2007, Armién et al. 2007, Barbosa et al. 2007), além de Turbina cordata, também no Brasil (Dantas et al. 2006). Este trabalho relata a ocorrência da intoxicação por Sida carpinifolia (Malvaceae) em bovinos no Rio Grande do Sul e descreve os achados epidemiológicos, clínico-patológicos, lectina-histoquímicos e ultraestruturais associados com a doença.

\section{MATERIAL E MÉTODOS}

Foram analisados quatro surtos (A, B, C e D) em que cinco bovinos foram intoxicados naturalmente por Sida carpinifolia L.f. (fam. Malvaceae) no Rio Grande do Sul, no período 20012008. Os bovinos desse estudo foram numerados de 1 a 5 (Quadro 1). Dados epidemiológicos e clínicos foram obtidos pelos médicos veterinários das propriedades e pela equipe do Setor de Patologia Veterinária da Universidade Federal do Rio Grande do Sul (SPV-UFRGS), durante visita às propriedades. Foram realizadas quatro necropsias e, em um caso (Bovino 3), o material foi encaminhado ao SPV-UFRGS para exame histopatológico. Fragmentos de diversos órgãos foram coletados e fixados em formol $10 \%$, processados de forma rotineira para histologia e corados pela hematoxilina e eosina (HE). Fragmentos de tálamo do bovino 3 foram fixados em glutaraldeído e enviados para o Laboratório Regional de Diagnóstico (LRD) da Universidade Federal de Pelotas (UFPel) para exame de microscopia eletrônica de transmissão.

Fragmentos de SNC dos Bovinos 2, 3 e 5 e do gânglio celíaco do Bovino 3 foram submetidos à técnica de lectinahistoquímica (Goldstein \& Hayes 1978) com a utilização de lectinas obtidas comercialmente (Vector Laboratories, Burlingame, CA) (Quadro 2). As lâminas foram desparafinizadas em xilol e hidratadas em concentrações decrescentes de álcool. A inativação da peroxidase endógena foi feita com peróxido de hidrogênio $3 \%$, por 30 minutos e a recuperação, com calor e solução de tampão citrato $10 \mathrm{mM}$ (pH 6.0), em banho-maria $\left(100^{\circ} \mathrm{C}\right)$, por 15 minutos. Para a diminuição das ligações ines-

\section{Quadro 1. Dados gerais referentes aos bovinos intoxicados naturalmente por Sida carpinifolia no Rio Grande do Sul}

\begin{tabular}{cccccc}
\hline Bovino $\mathrm{n}^{\circ}$ & Surto & Ano & Raça & Sexo & Idade (anos) \\
\hline 1 & $\mathrm{~A}$ & 2001 & SRD & $\mathrm{F}$ & 0,8 \\
2 & $\mathrm{~B}$ & 2006 & Holandês & $\mathrm{F}$ & 5 \\
3 & $\mathrm{C}$ & 2008 & Holandês & $\mathrm{F}$ & 12 \\
4 & $\mathrm{C}$ & 2008 & Holandês & $\mathrm{F}$ & 0,8 \\
5 & $\mathrm{D}$ & 2008 & SRD & $\mathrm{M}$ & 1,5 \\
\hline
\end{tabular}

$\mathrm{SRD}=$ sem raça definida, $\mathrm{F}=$ fêmea; $M=$ macho.

\section{Quadro 2. Lectinas utilizadas no estudo histoquímico de intoxicação} natural por Sida carpinifolia em bovinos no Rio Grande do Sul

\begin{tabular}{lcc}
\hline \multicolumn{1}{c}{ Lectina } & Abreviatura & Especificidade Carbohidrato $^{\text {a }}$ \\
\hline Concanavalia ensiformis & Con-A & a-D-Man; a-D-Glc \\
Glycine Max & SBA & a-D-GalNac; b-D-GalNac; a e b-Gal \\
Dolichos biflorus & DBA & a-D-GalNac \\
Ulex europaeus-1 & UEA-1 & a-L-Fuc \\
Triticum vulgaris & WGA & b-D-GlcNac, NeuNac \\
Succinyl-WGA & sWGA & (b-(1-4)-D-GlcNac) ${ }^{2}$ \\
Arachis hypogaea & PNA & b-D-Gal(1-3) GalNac \\
Ricinus communis-1 & RCA-1 & b-D-Gal > a-D-Gal
\end{tabular}

a Goldstein \& Hayes (1978). Fuc = fucose; Gal = galactose; GalNac = N-acetilgalactosamina; Glc = glicose; GlcNac = N-acetil-glicosamina; Man = manose; NeuNac = ácido N-acetil-neuraminico. 
pecíficas (background), os cortes foram tratados com leite desnatado (MolicoO) $5 \%$, durante 30 minutos. Subsequentemente, as lectinas foram aplicadas na diluição de $5 \mathrm{mg} / \mathrm{ml}$ em PBS (phosphate buffered saline) $\mathrm{pH} 7.4$, exceto por Con-A que foi utilizada em $1 \mathrm{mg} / \mathrm{ml}$. Seguindo-se, as lâminas foram incubadas em câmara úmida a $4^{\circ} \mathrm{C}$ por $12-14$ horas overnight, lavadas individualmente com água destilada e PBS, tratadas com estreptavidina (DAKO LSAB 2 kit, DAKO Corp., Carpinteria, CA) por 20 minutos em câmara úmida. Os cortes foram revelados com diaminobenzidine (DAB) (DAKO) por 5 minutos ou até atingir coloração marrom e contracorados com hematoxilina de Harris. Por se tratar de doença com sinais clínicos nervosos, todos os casos foram submetidos à técnica de imunohistoquímica para raiva (Pedroso et al. 2008) e encefalopatia espongiforme transmissível (EET).

\section{RESULTADOS}

\section{Epidemiologia e sinais clínicos}

Nas propriedades visitadas, a pastagem nativa dos piquetes onde os bovinos eram mantidos constituía a principal fonte de alimento e apresentava infestações por Sida carpinifolia, às vezes, em matos de eucaliptos (Eucalyptus globulus) (Fig.1). Os surtos analisados (A, B, C e D) tiveram morbidade de $13 \%, 15 \%, 40 \%$ e $55,55 \%$, respectivamente.

Surto A. Pequena propriedade localizada em Triunfo/ $\mathrm{RS}$, onde de um total de quinze bovinos, um morreu e

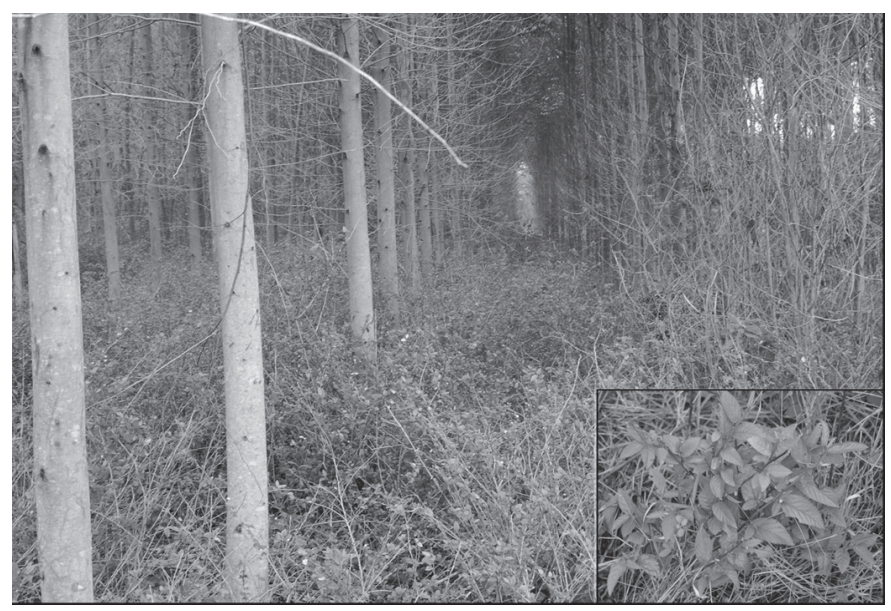

Fig.1. Infestação por Sida carpinifolia em mato de Eucaliptos (Eucalyptus globulus), Rio Grande do Sul. No detalhe, o aspecto de Sida carpinifolia.

outro desenvolveu doença caracterizada por emagrecimento, balanço e tremores da cabeça, além de quedas durante o andar. Esse bovino foi eutanasiado e necropsiado.

Surto B. Município de Triunfo/RS. De um total de vinte bovinos (4 vacas, 2 bois e 14 bezerros), adoeceram três vacas em final de gestação. Após apresentar-se subitamente em decúbito esternal com fratura no membro posterior direito (provavelmente associada com tentativas infrutíferas de manter a estação), uma vaca foi eutanasiada e necropsiada.

Surto C. Município de Caxias do Sul/RS. Propriedade com 160 hectares e um total de trinta bovinos. História de doze mortes (oito vacas e quatro bezerros) nos últimos quatro meses, com quadro clínico de emagrecimento, difi- culdade de permanecer em estação (Fig.2), incoordenação e quedas frequentes. Um bovino com esses sinais foi eutanasiado e fragmentos de fígado e cérebro do mesmo foram encaminhados ao SPV-UFRGS para análise histopatológica. Outro bovino, com doença semelhante e que foi eutanasiado e necropsiado, apresentou HR test (head

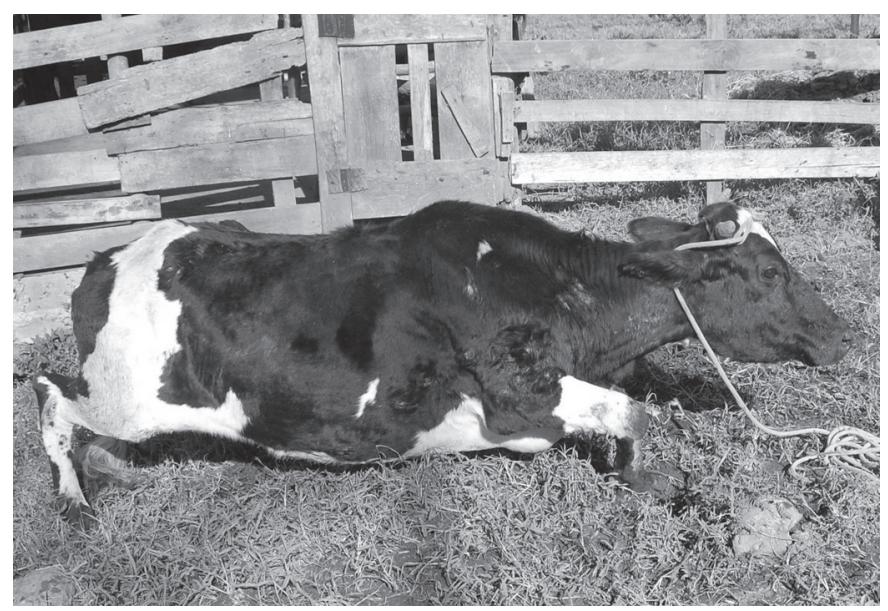

Fig.2. Intoxicação por Sida carpinifolia em bovinos no Rio Grande do Sul. Bovino $3 \mathrm{com}$ dificuldade de permanecer em estação após o "HR test".

raising test) positivo, o qual conforme Pienaar et al. (1976), consiste em queda subsequente à flexão da cabeça para cima e caudalmente seguida, após alguns segundos, de soltura rápida.

Surto D. Município de Triunfo/RS. De um total de nove bovinos, cinco haviam morrido no decorrer de seis meses, com sinais clínicos de emagrecimento, incoordenação e quedas ao movimento. Um bovino em decúbito lateral, movimentos de pedalagem, tremores e hiperexcitação, além de HR test positivo foi eutanasiado e necropsiado. Dados referentes ao ano de ocorrência, raça, sexo e idade dos bovinos estão apresentados no Quadro 1.

\section{Achados patológicos}

Não foram observadas alterações macroscópicas significativas. As principais alterações histológicas foram observadas no sistema nervoso central e incluíram vacuolização dos neurônios de Purkinje do cerebelo (Fig.3), núcleos do tronco encefálico e alguns neurônios na camada granular, além de esferóides axonais. No cerebelo dos cinco bovinos havia neurônios encolhidos, com núcleos picnóticos, citoplasma eosinofílico (Fig.4) e, muitas vezes, desaparecimento de neurônios de Purkinje. Em dois casos havia tumefação e vacuolização leve a moderada de núcleos da ponte e, em três casos, havia tumefação e vacuolização leve a moderada no mesencéfalo, na altura dos colículos rostrais. Na região do tálamo, havia leve tumefação de neurônios. Outras lesões consistiram de vacuolização das células acinares do pâncreas (2/3) (Fig.5), células foliculares da tireoide (1/3), células tubulares do rim (3/4) e hepatócitos (5/5). Em dois casos (Bovinos 1 e 3), verificou-se vacuolização leve a moderada de alguns 
neurônios do gânglio celíaco (2/2) e do plexo mioentérico (1/1), bem como do epitélio do epidídimo (1/1).

\section{Microscopia eletrônica e lectina-histoquímica}

No tálamo do bovino 3, havia vacúolos delimitados por membrana com conteúdo finamente granulado (Fig.6). Na lectina-histoquímica, havia marcações no SNC, com graus variáveis de leves a moderadas, especificamente no citoplasma dos neurônios de Purkinje, da camada granular (inclusive os de Golgi) e da camada molecular do cerebelo. Tais marcações foram mais evidentes quando tratadas com Concanavalia ensiformis (Con-A), Triticum vulgaris (WGA) (Fig.7) e Succinyl Triticum vulgaris (sWGA). Também houve marcação em neurônios do gânglio celíaco do Bovino 3 (Fig.8) e no telencéfalo. Não houve marcação para as outras lectinas.

\section{Imuno-histoquímica}

Todos os testes para raiva e EET foram negativos.

\section{DISCUSSÃO}

O diagnóstico da intoxicação por Sida carpinifolia nos bovinos foi baseado nos achados clínico-epidemiológicos,

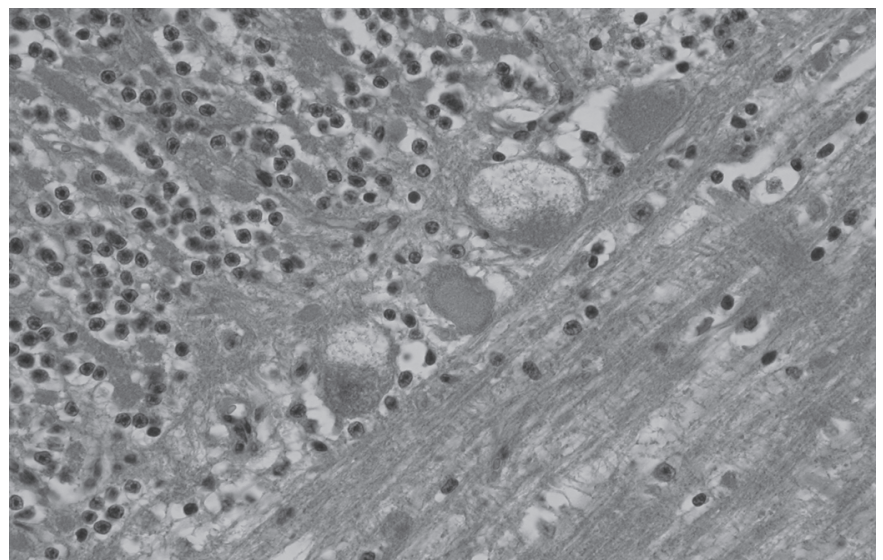

Fig.3. Vacuolização de neurônios de Purkinje do cerebelo, na intoxicação por Sida carpinifolia em bovinos no Rio Grande do Sul (Bovino 2). HE, obj.40x.

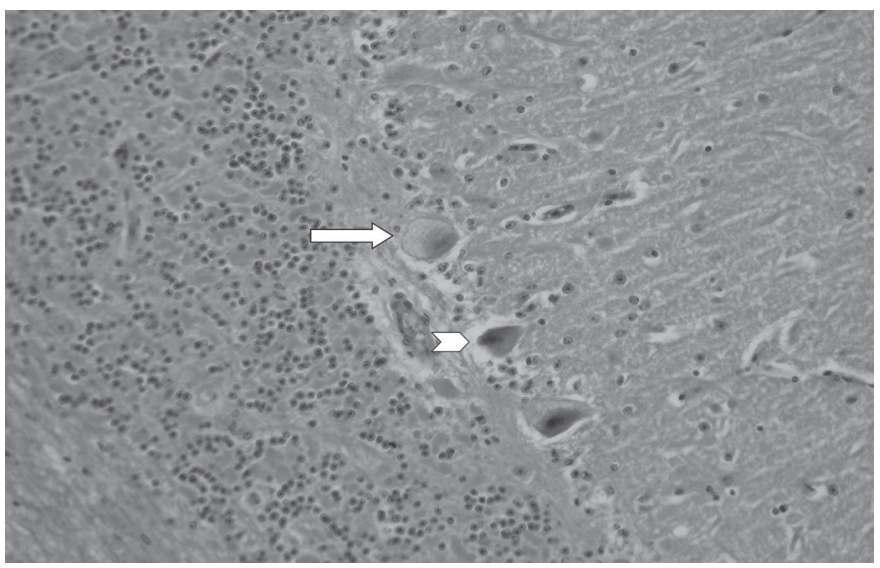

Fig.4. Neurônio vacuolizado (seta) e dois neurônios retraídos e eosinofílicos (cabeça de seta) no cerebelo, na intoxicação por Sida carpinifolia em bovinos no Rio Grande do Sul. (Bovino 2). HE, obj.20x.

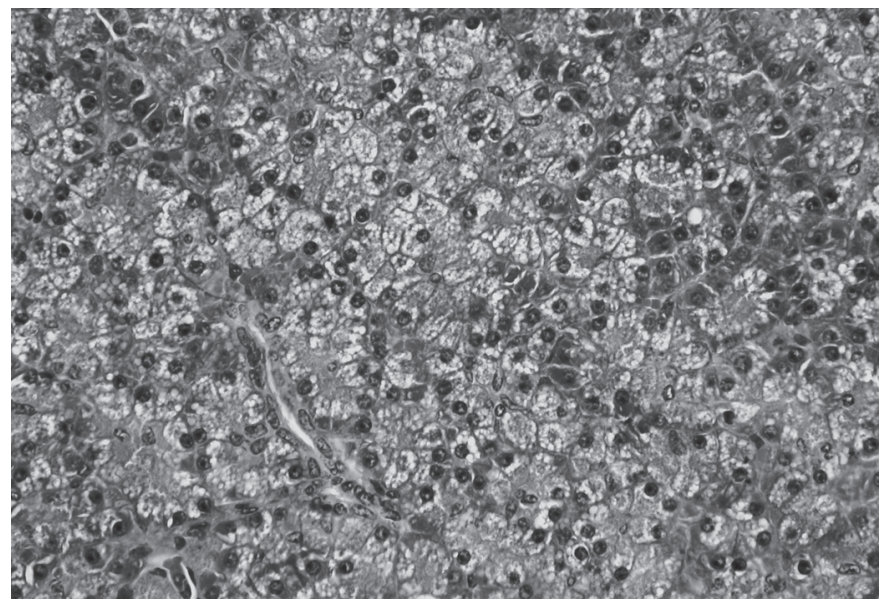

Fig.5. Vacuolização moderada das células acinares do pâncreas, na intoxicação por Sida carpinifolia em bovinos no Rio Grande do Sul (Bovino 5). HE, obj.40x.

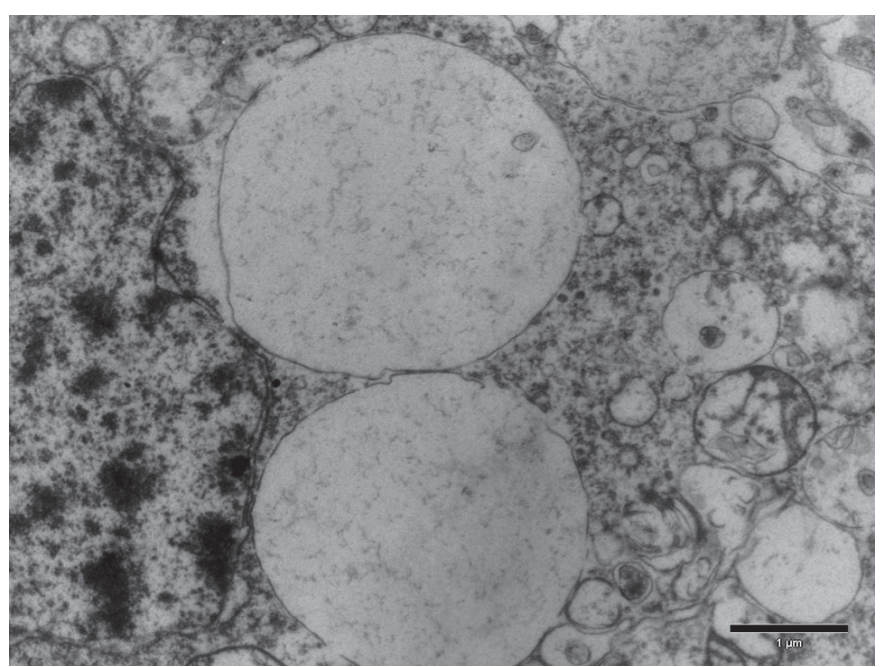

Fig.6. Vacúolos contendo material finamente granulado delimitado por membrana no tálamo, na intoxicação por Sida carpinifolia em bovinos no Rio Grande do Sul. (Bovino 3). Microscopia eletrônica de transmissão. Barra: $2 \mu \mathrm{m}$.

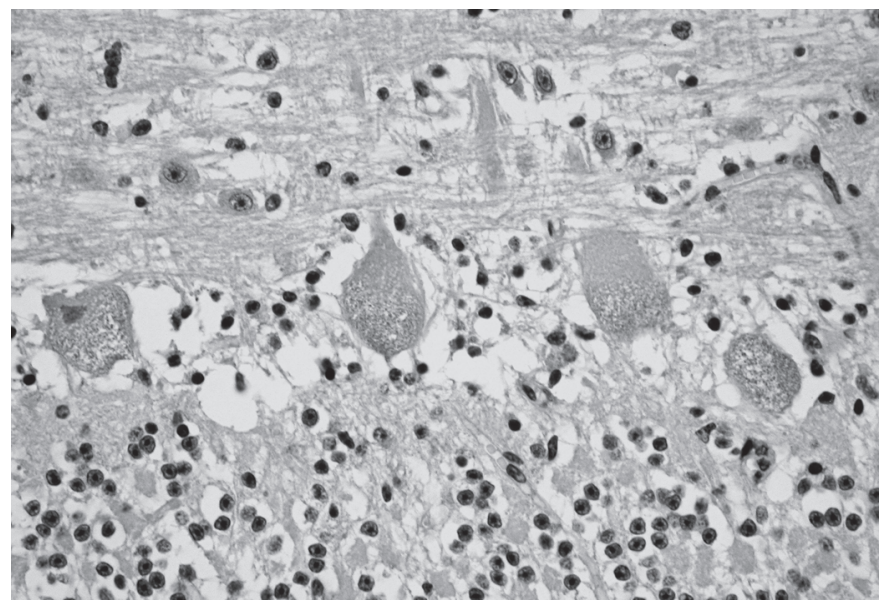

Fig.7. Intoxicação por Sida carpinifolia em bovinos no Rio Grande do Sul (Bovino 2). Marcação para Triticum vulgaris (WGA) em neurônio de Purkinje. Lectina-histoquímica, obj.40x. 


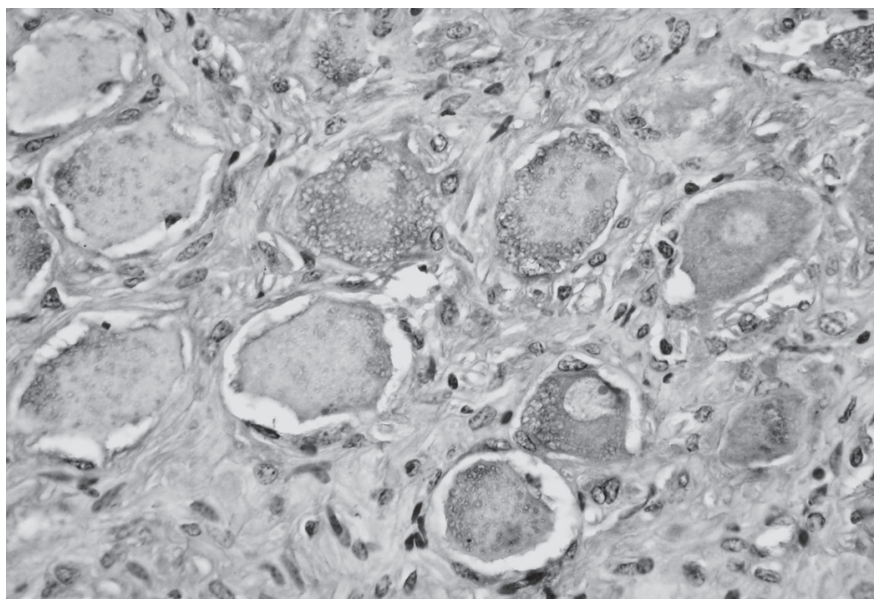

Fig.8. Intoxicação por Sida carpinifolia em bovinos no Rio Grande do Sul (Bovino 3). Marcação para Concanavalia ensiformis (Con-A) em neurônio do gânglio celíaco. Lectinahistoquímica, obj.40x.

além das alterações histológicas, ultraestruturais e lectinahistoquímicas características. Durante visitas às propriedades com casos da doença, constatou-se que S. carpinifolia era a vegetação predominante nos piquetes e, portanto, a principal fonte de alimentação dos bovinos. Essa situação foi idêntica àquelas observadas em casos de intoxicação natural em caprinos, ovinos e pôneis no Rio Grande do Sul (Colodel et al. 2002a, Loretti et al. 2003, Seitz et al. 2005a), como também em bovinos em Santa Catarina (Furlan et al. 2009). Segundo os proprietários da região de Triunfo/RS, há vários anos tem havido mortes de bovinos afetados por doença caraterizada por sinais nervosos e mantidos em áreas infestadas por $S$. carpinifolia e com pouca disponibilidade de forragem. Há também relatos de que os animais adquiriam avidez pela planta, mesmo quando outros alimentos estavam à disposição. Tais fatos também foram relatados previamente (Colodel et al. 2002a, Furlan et al. 2009).

Experimentalmente, demonstrou-se que doses de 10g/ kg por um período de 120 dias provocam alterações clínicas leves em bovinos; enquanto que 30 e $40 \mathrm{~g} / \mathrm{kg}$ administradas por um período de 150 dias produzem alterações neurológicas moderadas a acentuadas (Furlan et al. 2008). O quadro clínico em bovinos (caracterizado principalmente por incoordenação motora, tremores musculares, decúbito, movimentos de pedalagem e morte) foi semelhante ao observado nas intoxicações naturais em caprinos (Driemeier et al. 2000, Colodel et al. 2002a), ovinos (Seitz et al. 2005a) e bovinos (Furlan et al. 2009), como também ao observado na reprodução experimental em caprinos, ovinos e bovinos (Colodel et al. 2002a, Seitz et al. 2005b, Furlan et al. 2008). Em dois dos bovinos avaliados, observou-se teste HR positivo, pois animais com alterações neurológicas, especialmente cerebelares e vestibulares, demonstram incoordenação motora e ataques epileptiformes, quando submetidos ao procedimento (Pienaar et al. 1976) que, portanto, tem sido ferramenta diagnóstica auxiliar em casos de intoxicação por S. carpinifolia.
As principais alterações microscópicas observadas nos bovinos intoxicados naturalmente desse estudo foi distensão e vacuolização dos neurônios de Purkinje do cerebelo, além de vacuolização nas células foliculares da tireoide e células acinares do pâncreas. Essas alterações foram observadas em bovinos naturalmente intoxicados (Furlan et al. 2008, Furlan et al. 2009), como também em caprinos, pôneis, ovinos e cervídeos intoxicados naturalmente (Driemeier et al. 2000, Colodel et al. 2002a, Loretti et al. 2003, Seitz et al. 2005a, Pedroso et al. 2009). Solanum fastigiatum (jurubeba) (Mendez \& Riet-Correa, 2008) e Phalaris angusta (Gava et al. 1999) devem ser consideradas no diagnóstico diferencial de intoxicação por S. carpinifolia na Região Sul do Brasil, pois também causam quadro clínico neurológico. A intoxicação por Solanum fastigiatum apresenta sinais cerebelares caracterizados por crises periódicas do tipo epileptiforme, sendo que a duração varia de poucos segundos a um minuto. Nessa doença, há vacuolização celular limitada aos neurônios de Purkinje do cerebelo (Rech et al. 2006, Mendéz \& Riet-Correa, 2008). A técnica de lectina-histoquímica permite a diferenciação, pois a intoxicação por $S$. carpinifolia se caracteriza como uma $\alpha$ manosidase (Driemeier et al. 2000, Seitz et al. 2005a) e a intoxicação por S. fastigiatum, como uma doença de depósito glicolipídico (Paulovich et al. 2002). Na intoxicação por Phalaris angusta, evidencia-se macroscopicamente uma coloração verde-azulada nas regiões do tálamo, mesencéfalo e medula oblonga e, na microscopia, há pigmento granular marrom-amarelado no pericário de neurônios que não é observado na intoxicação por S. carpinifolia (Souza \& Irigoyen 1999).

A histoquímica com lectinas tem se mostrado uma ferramenta importante para o diagnóstico de doenças de depósito lisossomal, incluindo as induzidas por plantas tóxicas. $\mathrm{Na}$ á-manosidose, há acúmulo de oligossacarídeos contendo resíduos $\alpha$-manose e $\beta$-N-acetil-glicosamina (GlcNAc) (Beaudet 1983). Todos os casos analisados demonstraram marcações para lectinas Con-A, sWGA e WGA. A lectina Con-A se liga mais especificamente à $\alpha$-manose (Goldstein \& hayes, 1978) e as lectinas sWGA e WGA marcam os resíduos GlcNAc (Monsingny et al. 1980). Esses são os primeiros registros de intoxicação natural por $S$. carpinifolia em bovinos no Rio Grande do Sul. Por se encontrar disseminada no Rio Grande do Sul, ressalta-se a importância do diagnóstico de intoxicação por Sida carpinifolia em herbívoros no estado. Após o diagnóstico, os proprietários transferiram os demais bovinos dos locais infestados pela planta para outros locais livres de $S$. carpinifolia e não houve registros de novos casos da intoxicação.

Agradecimentos.- Aos colegas e funcionários do Setor de Patologia Veterinária da UFRGS pela valiosa ajuda. Parte deste trabalho foi financiadao pelo Conselho Nacional de Desenvolvimento Científico e Tecnológico (CNPq).

\section{REFERÊNCIAS}

Agamanolis D.P. 1995. The pathology of lysosomal storage diseases. Pathol. Annu. 30:247-285.

Antoniassi N.A.B., Ferreira E.V., Santos C.E.P., Arruda L.P., Campos J.L.E., Nakazato L. \& Colodel E.M. 2007. Intoxicação espontânea 
por Ipomoea carnea subsp. fistulosa (Convolvulaceae) em bovinos no Pantanal Matogrossense. Pesq. Vet. Bras. 27:415-418.

Armestro R.R., Repetto A.E., Ortega H.H., Peralta C.J., Pensiero J.F., Rei P.K. \& Salverti N.R. 2004. Intoxicacion em cabras por ingestion de Ipomoea hieronymi var. caichaquina en la província de Catamarca, Argentina. Vet. Arg. 21:232-241.

Armién A.G., Tokarnia C.H., Peixoto P.V. \& Frese K. 2007. Spontaneous and experimental glycoprotein storage disease of goats induced by Ipomoea carnea subsp. fistulosa (Convolvulaceae). Vet. Pathol. 44:170-184.

Balogh K.I.M., Dimande A.P., Van der Lugt J.J., Molyneux R.J., Naudé T.W. \& Welman W.G. 1999. A lysosomal storage disease induced by Ipomoea carnea in goats in Mozambique. J. Vet. Invest. 11:266-273.

Barbosa R.C., Riet-Correa F., Lima E.F., Medeiros R.M.T., Guedes K.M.R., Gardner D.R., Molyneux R.J. \& Melo L.E.H. 2007. Experimental swainsonine poisoning in goats ingesting Ipomoea sericophylla and Ipomoea riedelli (Convolvulaceae). Pesq. Vet. Bras. 27:409-414.

Barbosa R.C., Riet-Correa F., Medeiros R.M.T., Lima E.F., Barros S.S., Gimeno E.J., Molyneux R.J. \& Gardner D.R. 2006. Intoxication by Ipomoea sericophylla and Ipomoea riedelli in goats in the state of Paraíba, northeastern Brazil. Toxicon 47:371-379.

Bedin M., Colodel E.M., Giugliani R., Zlotowski P., Cruz C.E.F. \& Driemeier D. 2009. Urinary oligosaccharides: A peripheral marker for Sida carpinifolia exposure or poisoning. Toxicon 53:591-594.

Beaudet A.L. 1983. Disorders of glycoprotein degradation: Mannosidosis, fucosidosis, sialisidosis ad aspartylglycosaminuria, p.788-802. In: Stanbury J.B., Wyngaarden J.B., Fredrickson D.A.S., Goldstein J.L. \& Brown M.S. (Eds), The Metabolic Basis of Inherited Diseases. $5^{\text {th }}$ ed. McGraw-Hill, New York.

Colegate S.M., Dorling P.R. \& Huxtable CR. 1979. A spectroscopic investigation of swainsonine: An alfa-mannosidase inhibitor isolated from Swainsona canescens. Aust. J. Chem. 32:2257-2264.

Colodel E.M., Driemeier D., Loretti A.P., Gimeno E.J., Traverso S.D., Seitz A.L. \& Zlotowski P. 2002a. Aspectos clínicos e patológicos da intoxicação por Sida carpinifolia (Malvaceae) em caprinos no Rio Grande do Sul. Pesq. Vet. Bras. 22:51-57.

Colodel E.M., Gardner D.R., Zlotowski P. \& Driemeier D. 2002b. Identification of Swainsonine as a glycoside inhibitor responsible for Sida carpinifolia poisoning. Vet. Hum. Toxicol. 44:177-178.

Damir H.A., Adam S. \& Tartour G. 1987. The effects of Ipomoea carnea on goats and sheep. Vet. Hum. Toxicol. 29:316-319.

Dantas A.F.M., Riet-Correa F., Gardner D.R., Medeiros R.M.T., Barros S.S., Anjos B.L. \& Lucena R.B. 2007. Swainsonine-induced lysosomal storage disease in goats caused by the ingestion of Turbina cordata in Northeastern Brazil. Toxicon 49:111-116.

Driemeier D., Colodel E.M., Gimeno E.J. \& Barros S.S. 2000. Lysosomal storage disease caused by Sida carpinifolia in goats. Vet. Pathol. 37:153-159.

Furlan F.H., Lucioli J., Veronezi L.O., Traverso S.D. \& Gava A. 2008. Intoxicação experimental por Sida carpinifolia (Malvaceae) em bovinos. Pesq. Vet. Bras. 28:57-62.

Furlan F.H., Lucioli J., Veronezi L.O., Medeiros A., Barros S.S., Traverso S.D. \& Gava A. 2009. Spontaneous lysosomal storage disease caused by Sida carpinifolia (Malvaceae) poisoning in cattle. Vet. Pathol. 46:343-347.

Gava A., Sousa R.S., de Deus M.S., Pilati C., Cristani J., Mori A.M. \& Neves D.S. 1999. Phalaris angusta (Gramineae) como causa de enfermidade neurológica em bovinos no Estado de Santa Catarina. Pesq. Vet. Bras. 19:35-38.

Godoy G.S., Castro Netto A., Momo C., Dune A.C., Ávila L.G., Alessi A.C., Marques L.C. \& Castro M.B. 2005. Intoxicação natural por Sida carpinifolia (Malvaceae) em caprinos no estado de São Paulo. Arq. Bras. Med. Vet. Zootec. 57(Supl.1):25.
Goldstein I.J. \& Hayes C.E. 1978. The lectins carbohydrate binding proteins of plants and animals. Adv. Carbohydr. Chem. Biochem. 35:127-340.

Kissmann K.G. \& Groth D.G. 2000. Plantas Infestantes e Nocivas. Tomo III. BASF S.A., São Bernardo do Campo, SP. 722p.

Lorenzi H. 2000. Plantas daninhas do Brasil: Terrestres, aquáticas, parasitas, tóxicas e medicinais. $5^{\underline{a}}$ ed. Plantarum Ltda, Nova Odessa, SP. 339p.

Loretti A.L.P., Colodel E.M., Gimeno E.J. \& Driemeier. 2003. Lysosomal storage disease in Sida carpinifolia toxicosis: an induced mannosidosis in horses. Equine Vet. J. 35:434-438.

Méndez M. del C. \& Riet-Correa F. 2008. Plantas Tóxicas e Micotoxicoses. Editora e Gráfica Universitária, Pelotas, RS. 298p.

Molyneux R.J. \& James L.F. 1982. Loco intoxication: Indolizidine alkaloids of spotted locoweed (Astragalus lentiginosus). Science 216:190191.

Monsigny M., Roche A.C., Sene C., Marget-Domma R. \& Delmotte F. 1980. Sugar-lectin interations: How does wheat-germ agglutinin bind sialoglycoconjugates? Eur. J. Biochem. 104:147-153.

Moremen K.W. 2002. Golgi $\alpha$-manosidase II deficiency in vertebrate systems: implications for asparagines-linked oligosaccharide processing in mammals. Biochem. Biophys. Acta. 1573:225-235.

Paganini Filho W.S., Tirapelli A.C.N., Pereira T.G., Wouters A.T.B. \& Wouters F. 2008. Intoxicação por Sida carpinifolia em ovinos. Encontro Nacional de Diagnóstico Veterinário, 28 jun.-3 jul., Campo Grande, MS. (Resumo)

Paulovich F.B., Portiansky E.L., Gimeno E.J., Schild A.L., Méndez M. del C. \& Riet-Correa F. 2002. Lectin histochemical study of lipopigments present in the cerebellum of Solanum fastigiatum var. fastigiatum intoxicated cattle. J. Vet. Med. A. 49:473-477.

Pedroso P.M.O., Pescador C.A., Bandarra P.M., Raymundo D.L., Borba M.R., Wouters F., Bezerra Júnior P.S. \& Driemeier D. 2008. Padronização da técnica de imuno-histoquímica para raiva em amostras de sistema nervoso central de bovinos fixados em formol e emblocados em parafina. Pesq. Vet. Bras. 28:627-632.

Pedroso P.M.O., Von Hohendorf R., Oliveira L.G.S., Schmitz M., Cruz C.E.F., Driemeier D. 2009. Sida carpinifolia (Malvaceae) poisoning in fallow deer (Dama dama). J. Zoo Wildl. Med. 40:583-585.

Pfister J.A., Stegelmeier B.L., Gardner D.R. \& James L.F. 2003. Grazing of spotted locoweed (Astragalus lentiginosus) by cattle and horses in Arizona. J. Anim. Sci. 81:2285-2293.

Pienaar F.M., Kellerman T.S., Basson P.A., Jenkins W.L. \& Vahrmejer J. 1976. Maldronksiekte in cattle: A neuronopathy caused by Solanum kwebense N.E.Br. Onderstepoort J. Vet. Res. 43:67-74.

Rech R.R., Rissi D.R., Rodrigues A., Pierezan F., Piazer J.V.M., Kommers G.D. \& Barros C.S.L. 2006. Intoxicação por Solanum fastigiatum (Solanaceae) em bovinos: epidemiologia, sinais clínicos e morfometria das lesões cerebelares. Pesq. Vet. Bras. 26:183-189.

Ríos E., Cholich L., Silva J. \& Acosta de Pérez O. 2008. Histopathological lesions in central nervous system of goats poisoned by Ipomoea carnea. Revta Vet. 19:130-134.

Seitz A.L., Colodel E.M., Schmitz M., Gimeno E.J. \& Driemeier D. 2005a. Use de lectin histochemistry to diagnose Sida carpinifolia (Malvaceae) poisoning in sheep. Vet. Rec. 156:386-388.

Seitz A.L., Colodel E.M., Barros S.S. \& Driemeier D. 2005b. Intoxicação experimental por Sida carpinifolia (Malvaceae) em ovinos. Pesq. Vet. Bras. 25:15-20.

Sousa R.S. \& Irigoyen L.F. 1999. Intoxicação experimental por Phalaris angusta (Gramineae) em bovinos. Pesq. Vet. Bras. 19:116-122.

Stegelmeier B.L., Molyneux R.J., Elbein A.D. \& James L.F. 1995. The lesions of locoweed (Astragalus mollissimus), swainsonine, and castanospermine in rats. Vet. Pathol. 32:289-298. 